\title{
Singular limiting solutions for elliptic problem involving exponentially dominated nonlinearity and convection term
}

Sami Baraket ${ }^{1 *}$, Imed Abid ${ }^{2}$, Taieb Ouni ${ }^{2}$ and Nihed Trabelsi ${ }^{2}$

* Correspondence:
smbaraket@yahoo.fr
'Department of Mathematics,
College of Science, King Saud
University, P.O. Box 2455, Riyadh
11451, Saudi Arabia
Full list of author information is
available at the end of the article

* Correspondence: smbaraket@yahoo.fr College of Science, King Saud University, P.O. Box 2455, Riyadh Full list of author information is

\begin{abstract}
Given $\Omega$ bounded open regular set of $\mathbb{R}^{2}$ and $x_{1}, x_{2}, \ldots, x_{m} \in \Omega$, we give a sufficient condition for the problem

$$
-\operatorname{div}\left(e^{\lambda u} \nabla u\right)=\rho^{2} f(u)
$$

to have a positive weak solution in $\Omega$ with $u=0$ on $\partial \Omega$, which is singular at each $x_{i}$ as the parameters $\rho, \lambda>0$ tend to 0 and where $f(u)$ is dominated exponential nonlinearities functions.

2000 Mathematics Subject Classification: 35J60; 53C21; $58 \mathrm{~J} 05$.

Keywords: singular limits, Green's function, nonlinear Cauchy-data matching method
\end{abstract}

\section{Introduction and statement of the results}

We consider the following problem

$$
\left\{\begin{array}{rlll}
-\operatorname{div}(a(u) \nabla u) & =\rho^{2} f(u) & & \text { in } \quad \Omega \\
u & =0 & & \text { on } \partial \Omega,
\end{array}\right.
$$

where $\nabla$ is the gradient and $\Omega$ is an open smooth bounded subset of $\mathbb{R}^{2}$. The function $a$ is assumed to be positive and smooth. In the following, we take $a(u)=e^{\lambda u}$ and $f$ $(u)=e^{\lambda u}\left(e^{u}+e^{\gamma u}\right)$, for $\lambda>0$ and $\gamma \in(0,1)$, then problem (1) take the form

$$
\left\{\begin{array}{rlll}
-\Delta u-\lambda|\nabla u|^{2} & =\rho^{2}\left(e^{u}+e^{\gamma u}\right) \text { in } & \Omega \subset \mathbb{R}^{2} \\
u & =0 & \text { on } & \partial \Omega
\end{array}\right.
$$

Using the following transformation

$$
w=\left(\lambda \rho^{2} e^{u}\right)^{\lambda},
$$

then the function $w$ satisfies the following problem

$$
\left\{\begin{aligned}
-\Delta w & =w^{\frac{\lambda+1}{\lambda}}+\varrho w^{\frac{\gamma-1}{\lambda}} & & \text { in } \Omega \subset \mathbb{R}^{2} \\
w & =\left(\lambda \rho^{2}\right)^{\lambda} & & \text { on } \partial \Omega .
\end{aligned}\right.
$$


with $\varrho=\left(\lambda \rho^{2}\right)^{1-\lambda}$. So when $\lambda \rightarrow 0^{+}$, the exponent $q=\frac{\lambda+1}{\lambda}$ tends to infinity while the exponent $\frac{\gamma-1}{\lambda}$ tends to $-\infty$. For $\varrho \equiv 0$, problem (3) has been studied by Ren and Wei in [1]. See also [2].

We denote by $\varepsilon$ the smallest positive parameter satisfying

$$
\rho^{2}=\frac{8 \varepsilon^{2}}{\left(1+\varepsilon^{2}\right)^{2}} .
$$

Remark that $\rho \sim \varepsilon$ as $\varepsilon \rightarrow 0$. We will suppose in the following

$$
\left(A_{\lambda}\right): \quad \text { If } 0<\varepsilon<\lambda \text {, then } \quad \lambda^{1+\delta / 2} \varepsilon^{-\delta} \rightarrow 0 \quad \text { as } \lambda \rightarrow 0 \text {, for any } \delta \in(0,1) \text {. }
$$

In particular, if we take $\lambda=\mathcal{O}\left(\varepsilon^{2 / 3}\right)$, then the condition $\left(A_{\lambda}\right)$ is satisfied. Under the assumption $\left(A_{\lambda}\right)$, we can treat equation (2) as a perturbation of the following:

$$
-\Delta u=\rho^{2}\left(e^{u}+e^{\gamma u}\right) \quad \text { in } \Omega \subset \mathbb{R}^{2}
$$

for $\gamma \in(0,1)$.

Our question is: Does there exist $v_{\varepsilon, \lambda}$ a sequence of solutions of (2) which converges to some singular function as the parameters $\varepsilon$ and $\lambda$ tend to 0 ?

In [3], Baraket et al. gave a positive answer to the above question for the following problem

$$
\left\{\begin{aligned}
-\Delta u-\lambda|\nabla u|^{2} & =\rho^{2} e^{u} \text { in } \Omega \\
u & =0 \quad \text { on } \partial \Omega,
\end{aligned}\right.
$$

with a regular bounded domain $\Omega$ of $\mathbb{R}^{2}$. They give a sufficient condition for the problem (5) to have a weak solution in $\Omega$ which is singular at some points $\left(x_{i}\right)_{1 \leq i \leq m}$ as $\rho$ and $\lambda$ a small parameters satisfying $\left(A_{\lambda}\right)$, where the presence of the gradient term seems to have significant influence on the existence of such solutions, as well as on their asymptotic behavior.

In case $\lambda=0$ the authors in [4] gave also a positive answer for the following problem

$$
\left\{\begin{aligned}
-\Delta u & =\rho^{2}\left(e^{u}+e^{\gamma u}\right) & & \text { in } \Omega \subset \mathbb{R}^{2} \\
u & =0 & & \text { on } \partial \Omega,
\end{aligned}\right.
$$

for $\gamma \in(0,1)$ as $\rho$ tends to 0 . When $\lambda=0$ and $\gamma=0$, problem (2) reduce to

$$
\left\{\begin{aligned}
-\Delta u & =\rho^{2} e^{u} \text { in } \Omega \subset \mathbb{R}^{2} \\
u & =0 \quad \text { on } \partial \Omega .
\end{aligned}\right.
$$

The study of this problem goes back to 1853 when Liouville derived a representation formula for all solutions of (7) which are defined in $\mathbb{R}^{2}$, see [5]. It turns out that, beside the applications in geometry, elliptic equations with exponential nonlinearity also arise in modeling many physical phenomenon, such as thermionic emission, isothermal gas sphere, gas combustion, and gauge theory [6]. When $\rho$ tends to 0 , the asymptotic behavior of nontrivial branches of solutions of (7) is well understood thanks to the pioneer work of Suzuki [7] which characterizes the possible limit of nontrivial branches of solutions of (7). His result has been generalized in [8] to (6) with $\gamma<\frac{1}{4}$, and finally by Ye in [9] to any exponentially dominated nonlinearity $f(u)$. The existence of nontrivial branches of solutions with single singularity was first proved by Weston [10] and then a general result has been obtained by Baraket and Pacard [11]. These results were also 
extended, applying to the Chern-Simons vortex theory in mind, by Esposito et al. [12] and Del Pino et al. [13] to handle equations of the form $-\Delta u=\rho^{2} V(x) e^{u}$ where $V$ is a nonconstant positive potential. See also [14-16] wherever this rule is applicable. where the Laplacian is replaced by a more general divergence operator and some new phenomena occur. Let us also mention that the construction of nontrivial branches of solutions of semilinear equations with exponential nonlinearities allowed Wente to provide counter examples to a conjecture of Hopf [17] concerning the existence of compact (immersed) constant mean curvature surfaces in Euclidean space. Another related problem is the higher dimension problem with exponential nonlinearity. For example, the 4-dimensional semilinear elliptic problem with bi-Laplacian is treated in [18] and the problem with an additional singular source term given by Dirac masses is treated in [19] in the radial case. The results in $[18,19]$ are generalized to noncritical points of the reduced function, see [20].

We introduce now the Green's function $G\left(x, x^{\prime}\right)$ defined on $\Omega \times \Omega$, to be solution of

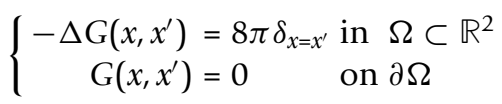

and let $H\left(x, x^{\prime}\right)=G\left(x, x^{\prime}\right)+4 \log \left|x-x^{\prime}\right|$, its regular part. Let $m \in \mathbb{N}$, we set

$$
\mathcal{F}\left(x_{1}, \ldots, x_{m}\right)=\sum_{j=1}^{m} H\left(x_{j}, x_{j}\right)+\sum_{i \neq j} G\left(x_{i}, x_{j}\right)
$$

which is well defined in $(\Omega)^{m}$ for $x_{i} \neq x_{j}$ for $i \neq j$. Our main result is the following

Theorem 1 Given $\beta \in(0,1)$. Let $\Omega$ an open smooth bounded set of $\mathbb{R}^{2}, \lambda>0$ satisfying the condition $\left(A_{\lambda}\right), \gamma \in(0,1)$ and $S=\left\{x_{1}, \ldots x_{m}\right\} \subset \Omega$ be a nonempty set. Assume that, the point $\left(x_{1}, \ldots, x_{m}\right)$ is a nondegenerate critical point of the function

$$
\mathcal{F}\left(x_{1}, \ldots, x_{m}\right)=\sum_{j=1}^{m} H\left(x_{j}, x_{j}\right)+\sum_{i \neq j} G\left(x_{i}, x_{j}\right) \quad \text { in }(\Omega)^{m},
$$

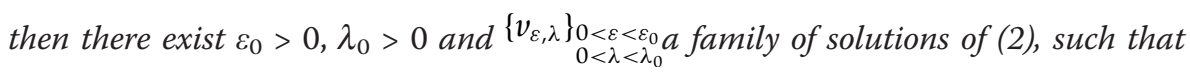

$$
\lim _{\substack{\varepsilon \rightarrow 0 \\ \lambda \rightarrow 0}} v_{\varepsilon, \lambda}=\sum_{j=1}^{m} G\left(x_{j}, \cdot\right) \quad \text { in } \mathcal{C}_{l o c}^{2, \beta}\left(\Omega-\left\{x_{1}, \ldots, x_{m}\right\}\right) .
$$

One of the purpose of the present paper is to present a rather efficient method: nonlinear Cauchy-data matching method to solve such singularly problems. This method has already been used successfully in geometric context (constant mean curvature surfaces, constant scalar curvature metrics, extremal Kähler metrics, manifolds with special holonomy, ...) and appeared in the study [18] in the context of partial differential equations.

\section{Construction of the approximate solution}

We first describe the rotationally symmetric approximate solutions of

$$
-\Delta u-\lambda|\nabla u|^{2}=\rho^{2}\left(e^{u}+e^{\gamma u}\right)
$$

in $\mathbb{R}^{2}$ which will play a central role in our analysis. Given $\varepsilon>0$, we define

$$
u_{\varepsilon}(x):=2 \log \left(1+\varepsilon^{2}\right)-2 \log \left(\varepsilon^{2}+|x|^{2}\right)
$$


which is clearly a solution of

$$
-\Delta u=\rho^{2} e^{u} .
$$

in $\mathbb{R}^{2}$. Let us notice that equations (11) is invariant under dilation in the following sense: If $v$ is a solution of (11) and if $\tau>0$, then $v(\tau \cdot)+2 \log \tau$ is also a solution of (11). With this observation in mind, we define for all $\tau>0$

$$
u_{\varepsilon, \tau}(x):=2 \log \left(1+\varepsilon^{2}\right)+2 \log \tau-2 \log \left(\varepsilon^{2}+|\tau x|^{2}\right) .
$$

\subsection{A linearized operator on $\mathbb{R}^{2}$}

For all $\varepsilon, \tau, \lambda>0$, we set

$$
R_{\varepsilon, \lambda}:=\tau r_{\varepsilon, \lambda} / \varepsilon \quad \text { where } \quad r_{\varepsilon, \lambda}:=\max \left(\varepsilon^{2(1-\gamma)-\delta / 2}, \sqrt{\lambda}, \sqrt{\varepsilon}\right) .
$$

for $\delta \in(0,1)$. We define the linear second order elliptic operator

$$
\mathbb{L}:=-\Delta-\frac{8}{\left(1+|x|^{2}\right)^{2}}
$$

which corresponds to the linearization of (11) about the solution $u_{1}\left(=u_{\varepsilon}=\tau=1\right)$ given by (10) which has been defined in the previous section. We are interested in the classification of bounded solutions of $\mathbb{L} w=0$ in $\mathbb{R}^{2}$. Some solutions are easy to find. For example, we can define

$$
\phi_{0}(x):=\frac{r}{2} \partial_{r} u_{1}(x)+1=2 \frac{1-r^{2}}{1+r^{2}},
$$

where $r=|x|$. Clearly $\mathbb{L} \phi_{0}=0$ and this reflects the fact that (11) is invariant under the group of dilations $\tau \rightarrow u(\tau \cdot)+2 \log \tau$. We also define, for $i=1,2$

$$
\phi_{i}(x):=-\partial_{x_{i}} u_{1}(x)=\frac{2 x_{i}}{1+|x|^{2}},
$$

which are also solutions of $\mathbb{L} \phi_{i}=0$. Since, these solutions correspond to the invariance of the equation under the group of translations $a \rightarrow u(\cdot+a)$. We recall the following result which classifies all bounded solutions of $\mathbb{L} w=0$ which are defined in $\mathbb{R}^{2}$.

Lemma $1[11]$ Any bounded solution of $\mathbb{L} w=0$ defined in $\mathbb{R}^{2}$ is a linear combination of $\varphi_{i}$ for $i=0,1,2$.

Let $B_{r}$ denote the ball of radius $r$ centered at the origin in $\mathbb{R}^{2}$.

Definition 1 Given $k \in \mathbb{N}, \beta \in(0,1)$ and $\mu \in \mathbb{R}$, we introduce the Hölder weighted spaces $\mathcal{C}_{\mu}^{k, \beta}\left(\mathbb{R}^{2}\right)$ as the space of functions $w \in \mathcal{C}_{\text {loc }}^{k, \beta}\left(\mathbb{R}^{2}\right)$ for which the following norm

$$
\left\|\left.w\right|_{\mathcal{C}_{\mu}^{k, \beta}\left(\mathbb{R}^{2}\right)}:=\right\| w \|_{\mathcal{C}^{k, \beta}\left(\bar{B}_{1}\right)}+\sup _{r \geq 1}\left(\left(1+r^{2}\right)^{-\mu / 2}\|w(r \cdot)\|_{\mathcal{C}_{\mu}^{k, \beta}\left(\bar{B}_{1}-B_{1 / 2}\right)}\right)
$$

is finite.

We define also

$$
\mathcal{C}_{\mathrm{rad}, \mu}^{k, \beta}\left(\mathbb{R}^{2}\right)=\left\{f \in \mathcal{C}_{\mu}^{k, \beta}\left(\mathbb{R}^{2}\right) ; \text { such that } f(x)=f(|x|), \forall x \in \mathbb{R}^{2}\right\} .
$$

As a consequence of the result of Lemma 1 , we recall the surjectivity result of $\mathbb{L}$ given in [11]. 


\section{Proposition 1 [11]}

(i) Assume that $\mu>1$ and $\mu \notin \mathbb{N}$, then

$$
\begin{array}{ccc}
L_{\mu}: C_{\mu}^{2, \beta}\left(\mathbb{R}^{2}\right) & \rightarrow & \mathcal{C}_{\mu-2}^{0, \beta}\left(\mathbb{R}^{2}\right) \\
w & \mapsto & \mathbb{L} w
\end{array}
$$

is surjective.

(ii) Assume that $\delta>0$ and $\delta \notin \mathbb{N}$ then

$$
\begin{array}{clc}
L_{\delta}: \mathcal{C}_{\mathrm{rad}, \delta}^{2, \beta}\left(\mathbb{R}^{2}\right) & \rightarrow & \mathcal{C}_{\mathrm{rad}, \delta-2}^{0, \beta}\left(\mathbb{R}^{2}\right) \\
w & \mapsto & \mathbb{L} w
\end{array}
$$

is surjective.

We set $\bar{B}_{1}^{*}=\bar{B}_{1}-\{0\}$, we define

Definition 2 Given $k \in \mathbb{N}, \beta \in(0,1)$ and $\mu \in \mathbb{R}$, we introduce the Hölder weighted spaces $\mathcal{C}_{\mu}^{k, \beta}\left(\bar{B}_{1}^{*}\right)$ as the space of functions in $\mathcal{C}_{\text {loc }}^{k, \beta}\left(\bar{B}_{1}^{*}\right)$ for which the following norm

$$
\|u\|_{\mathcal{C}_{\mu}^{k, \beta}\left(\bar{B}_{1}^{*}\right)}=\sup _{r \leq 1 / 2}\left(r^{-\mu}\|u(r \cdot)\|_{\mathcal{C}^{k, \beta}\left(\bar{B}_{2}-B_{1}\right)}\right),
$$

is finite.

Then, we define the subspace of radial functions in $\mathcal{C}_{\mathrm{rad}, \delta}^{k, \beta}\left(\bar{B}_{1}^{*}\right)$ by

$$
\mathcal{C}_{\mathrm{rad}, \delta}^{k, \beta}\left(\bar{B}_{1}^{*}\right)=\left\{f \in \mathcal{C}_{\delta}^{k, \beta}\left(\mathbb{R}^{2}\right) \text {; such that } f(x)=f(|x|), \forall x \in \bar{B}_{1}^{*}\right\} .
$$

We would like to find a solution $u$ of

$$
\Delta u+\lambda|\nabla u|^{2}+\rho^{2}\left(e^{u}+e^{\gamma u}\right)=0
$$

in $B_{\varepsilon_{\varepsilon}, \lambda}$. By using the transformation, $v(x)=u\left(\frac{\varepsilon}{\tau} x\right)+4 \log \varepsilon-2 \log \left(\tau\left(1+\varepsilon^{2}\right) / 2\right)$, then Eq. (15) is equivalent to

$$
\Delta v+\lambda|\nabla v|^{2}+2\left(e^{v}+\frac{2^{2(1-\gamma)} \varepsilon^{4(1-\gamma)}}{\left(\left(1+\varepsilon^{2}\right) \tau\right)^{2(1-\gamma)}} e^{\gamma v}\right)=0
$$

in $\bar{B}_{R_{\varepsilon, \lambda}}$. We look for a solution of (16) of the form $v(x)=u_{1}(x)+h(x)$, this amounts to solve

$$
\mathbb{L} h:=\Re(h)=\frac{8}{\left(1+|x|^{2}\right)^{2}}\left(e^{h}-h-1\right)-\frac{8 \varepsilon^{4(1-\gamma)}}{\left(\left(1+\varepsilon^{2}\right) \tau\right)^{2(1-\gamma)}\left(1+|x|^{2}\right)^{2 \gamma}} e^{\gamma h}+\lambda\left|\nabla\left(u_{1}+h\right)\right|^{2}
$$

In $\bar{B}_{R_{\varepsilon, \lambda}}$. We will need the following:

Definition 3 Given $\bar{r} \geq 1, k \in \infty, \beta \in(0,1)$ and $\mu \in \mathbb{R}$, the weighted space $\mathcal{C}_{\mu}^{k, \beta}\left(B_{\bar{r}}\right)$ is defined to be the space of functions $w \in \mathcal{C}^{k, \beta}\left(B_{\bar{r}}\right)$ endowed with the norm

$$
\left\|\left.w\right|_{\mathcal{C}_{\mu}^{k, \beta}\left(\bar{B}_{\bar{r}}\right)}:=\right\| w \|_{\mathcal{C}^{k, \beta}\left(B_{1}\right)}+\sup _{1 \leq r \leq \bar{r}}\left(r^{-\mu}\|w(r \cdot)\|_{\mathcal{C}^{k, \beta}\left(\bar{B}_{1}-B_{1 / 2}\right)}\right) .
$$

For all $\sigma \geq 1$, we denote by $\mathcal{E}_{\sigma}: \mathcal{C}_{\mu}^{0, \beta}\left(\bar{B}_{\sigma}\right) \rightarrow \mathcal{C}_{\mu}^{0, \beta}\left(\mathbb{R}^{2}\right)$ the extension operator defined by

$$
\varepsilon_{\sigma}(f)(x)=\left\{\begin{array}{cll}
f(x) & \text { for } & |x| \leq \sigma \\
\chi\left(\frac{|x|}{\sigma}\right) f\left(\sigma \frac{x}{|x|}\right) & \text { for } & |x| \leq \sigma,
\end{array}\right.
$$


where $t \alpha \chi(t)$ is a smooth non-negative cutoff function identically equal to 1 for $t \leq$ 1 and identically equal to 0 for $t \geq 2$. It is easy to check that there exists a constant $c$ $=c(\mu)>0$, independent of $\sigma \geq 1$, such that

$$
\left\|\varepsilon_{\sigma}(w)\right\|_{\mathcal{C}_{\mu}^{0, \beta}\left(\mathbb{R}^{2}\right)} \leq c|| w \|_{\mathcal{C}_{\mu}^{0, \beta}\left(\bar{B}_{\sigma}\right)} .
$$

We fix $\delta \in(0,1)$ and denote by $\mathcal{G}_{\delta}$ to be a right inverse of $\mathbb{L}_{\delta}$ provided by Proposition 1 . To find a solution of (17), it is enough to find a fixed point $h$, in a small ball of $\mathcal{C}_{\text {rad }, \delta}^{2, \beta}\left(\mathbb{R}^{2}\right)$, solution of

$$
h=\aleph(h)=\mathcal{G}_{\delta} \circ \mathcal{E}_{\delta} \circ \Re(h) .
$$

We have

$$
\Re(0)=\lambda\left|\nabla u_{1}\right|^{2}-\frac{8 \varepsilon^{4(1-\gamma)}}{\left(\left(1+\varepsilon^{2}\right) \tau\right)^{2(1-\gamma)}\left(1+|x|^{2}\right)^{2 \gamma}} .
$$

This implies that given $\kappa>0$, there exist $c_{\kappa}>0$ (only depend on $\kappa$ ), such that for $\delta \in$ $(0,1)$ and $|x|=r$, we have

$$
\begin{aligned}
\sup _{r \leq R_{\varepsilon, \lambda}} r^{2-\delta}|\Re(0)| & \leq \sup _{r \leq R_{\varepsilon, \lambda}} r^{2-\delta}\left(\lambda\left|\nabla u_{1}\right|^{2}+\frac{8 \varepsilon^{4(1-\gamma)}}{\left(\left(1+\varepsilon^{2}\right) \tau\right)^{2(1-\gamma)}\left(1+|x|^{2}\right)^{2 \gamma}}\right) \\
& \leq c_{\kappa}\left(\lambda+\max \left\{\varepsilon^{4(1-\gamma)}, \varepsilon^{2+\delta} r_{\varepsilon, \lambda}^{-2-\delta}\right\}\right) .
\end{aligned}
$$

Making use of Proposition 1 together with (19), we conclude that

$$
\|h\|_{\mathcal{C}_{\text {rad }, \delta}^{2, \beta}\left(\mathbb{R}^{2}\right)} \leq 2 c_{\kappa} r_{\varepsilon, \lambda}^{2} .
$$

Now, let $h_{1}, h_{2}$ such that $\left\|h_{i}\right\| \leq 2 c_{\kappa} r_{\varepsilon, \lambda}^{2}$ in $\mathcal{C}_{\text {rad }, \delta}^{2, \beta}\left(\mathbb{R}^{2}\right)$, then for $\delta \in(0,1-r]$ we have

$$
\begin{aligned}
& \sup _{r \leq R_{e, \lambda}} r^{2-\delta}\left|\Re\left(h_{2}\right)-\Re\left(h_{1}\right)\right| \\
& \leq c_{\kappa} \sup _{r \leq R_{\varepsilon, \lambda}} r^{2-\delta}\left(1+|x|^{2}\right)^{-2}\left|e^{h_{2}}-e^{h_{1}}+h_{1}-h_{2}\right|+c_{\kappa} \lambda \sup _{r \leq R_{\varepsilon, \lambda}} r^{2-\delta}\left(\left|\nabla\left(u_{1}+h_{2}\right)\right|^{2}-\left|\nabla\left(u_{1}+h_{1}\right)\right|^{2}\right) \\
& +c_{\kappa} \sup _{r \leq R_{\varepsilon, \lambda}} r^{2-\delta}\left(1+|x|^{2}\right)^{-2 \gamma}\left|e^{\gamma h_{1}}-e^{\gamma h_{2}}\right| \\
& \leq c_{\kappa} \max \left\{\varepsilon^{4(1-\gamma)}, \varepsilon^{2+\delta} r_{\varepsilon, \lambda}^{-2-\delta}\right\}\left\|h_{2}-h_{1}\right\|_{\mathcal{C}_{\text {rad, } \delta}^{2, \beta}\left(\mathbb{R}^{2}\right)}+c_{\kappa} \lambda\left\|h_{2}-h_{1}\right\|_{\mathcal{C}_{\text {rad }, \delta}^{2, \beta}\left(\mathbb{R}^{2}\right)}
\end{aligned}
$$

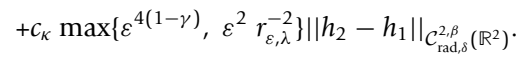

Similarly, making use of Proposition 1 together with condition $\left(A_{\lambda}\right)$ and (19), we conclude that given $\kappa>0$, there exist $\varepsilon_{\kappa}>0, \lambda_{\kappa}>0$ and $\bar{c}_{\kappa}>0$ (only depend on $\kappa$ ) such that

$$
\left\|\aleph\left(h_{2}\right)-\aleph\left(h_{1}\right)\right\|_{\mathcal{C}_{\mathrm{rad}, \delta}^{2, \beta}\left(\mathbb{R}^{2}\right)} \leq \bar{c}_{\kappa} r_{\varepsilon, \lambda}^{2}\left\|h_{2}-h_{1}\right\|_{\mathcal{C}_{\mathrm{rad}, \delta}^{2, \beta}\left(\mathbb{R}^{2}\right)} .
$$

Reducing $\lambda_{\kappa}>0$ and $\varepsilon_{\kappa}>0$ if necessary, we can assume that, $\bar{c}_{\kappa} r_{\varepsilon, \lambda}^{2} \leq \frac{1}{2}$ for all $\lambda \in$ $\left(0, \lambda_{\kappa}\right)$ and $\varepsilon \in\left(0, \varepsilon_{\kappa}\right)$. Then, (21) and (22) are enough to show that $h \mapsto \boldsymbol{N}$ is a contraction from $\left\{h \in \mathcal{C}_{\mathrm{rad}, \delta}^{2, \beta}\left(\mathbb{R}^{2}\right):\|h\|_{\mathcal{C}_{\text {rad }, \delta}^{2, \beta}\left(\mathbb{R}^{2}\right)} \leq 2 c_{\kappa} r_{\varepsilon, \lambda}^{2}\right\}$ into itself and hence has a unique fixed point $h$ in this set. This fixed point is solution of (20) in $\bar{B}_{R_{\varepsilon, \lambda}}$. We summarize this in the: 
Proposition 2 Given $\delta \in(0,1-\gamma]$ and $\kappa>1$, then there exist $\bar{c}_{\kappa}>0$ (independent of $\varepsilon$ and $\lambda)$ and a unique $h \in \mathcal{C}_{\text {rad, }, \delta}^{2, \beta}\left(\mathbb{R}^{2}\right)$ with $\|h\|_{\mathcal{C}_{\text {rad, }, \delta}^{2, \beta}\left(\mathbb{R}^{2}\right)} \leq 2 c_{\kappa} r_{\varepsilon, \lambda}^{2}$ such that

$$
v(x)=u_{1}(x)+h(x)
$$

solves (16) in $\bar{B}_{R_{\varepsilon, \lambda}}$.

\subsection{Analysis of the Laplace operator in weighted spaces}

In this section, we study the mapping properties of the Laplace operator in weighted Hölder spaces. Given $x_{1}, \ldots, x_{m} \in \Omega$, we define $\mathbf{x}:=\left(x_{1}, \ldots, x_{m}\right)$

$$
\bar{\Omega}^{*}(\mathbf{x}):=\bar{\Omega}-\left\{x_{1}, \ldots x_{m}\right\},
$$

and we choose $r_{0}>0$ so that the balls $B_{r_{0}}\left(x_{i}\right)$ of center $x_{i}$ and radius $r_{0}$ are mutually disjoint and included in $\Omega$. For all $r \in\left(0, r_{0}\right)$, we define

$$
\bar{\Omega}_{r}(\mathbf{x}):=\bar{\Omega}-\cup_{i=1}^{m} B_{r}\left(x_{i}\right) .
$$

With these notations, we have:

Definition 4 Given $k \in \mathbb{R}, \beta \in(0,1)$ and $v \in \mathbb{R}$, we introduce the Hölder weighted space $\mathcal{C}_{v}^{k, \beta}\left(\bar{\Omega}^{*}(\mathbf{x})\right)$ as the space of functions $w \in \mathcal{C}_{\text {loc }}^{k, \beta}\left(\bar{\Omega}^{*}(\mathbf{x})\right)$ for with the following norm

$$
\left\|\left.w\right|_{\mathcal{C}_{v}^{k, \beta}(\bar{\Omega} *(\mathbf{x}))}:=\right\| w \|_{\mathcal{C}^{k, \beta}\left(\bar{\Omega}_{r_{0 / 1}}\right)}+\sum_{i=1}^{m} \sup _{0<r \leq r_{0 / 2}}\left(r^{-v}\left\|w\left(x_{i}+r \cdot\right)\right\|_{\mathcal{C}^{k, \beta}\left(\bar{B}_{2}-B_{1}\right)}\right)
$$

is finite.

When $k \geq 2$, we denote by $\left[\mathcal{C}_{v}^{k, \beta}\left(\bar{\Omega}^{*}(\mathbf{x})\right)\right]_{0}$ be the subspace of functions $w \in \mathcal{C}_{v}^{k, \beta}\left(\bar{\Omega}^{*}(\mathbf{x})\right)$ satisfying $w=0$ on $\partial \Omega$. We recall the

Proposition 3 [21]Assume that $v<0$ and $v \notin \mathbb{Z}$, then

$$
\begin{array}{cccc}
\mathcal{L}_{v}: \quad\left[\mathcal{C}_{v}^{2, \beta}\left(\bar{\Omega}^{*}(\mathbf{x})\right)\right]_{0} & \rightarrow & \mathcal{C}_{v-2}^{0, \beta}\left(\bar{\Omega}^{*}(\mathbf{x})\right) \\
w & \mapsto & \Delta w
\end{array}
$$

is surjective. Denote by $\tilde{\mathcal{G}}_{v}$ a right inverse of $\mathcal{L}_{v}$.

Remark 1 Observe that, when $v<0, v \notin \mathbb{Z}$, the right inverse even though is not unique and can be chosen to depend smoothly on the points $x_{1}, \ldots, x_{m}$, at least locally. Once a right inverse is fixed for some choice of the points $x_{1}, \ldots, x_{m}$, a right inverse which depends smoothly on some points $\tilde{x}_{1}, \ldots, \tilde{x}_{m}$ close to $x_{1}, \ldots, x_{m}$ can be obtained using a simple perturbation argument. This argument will be used later in the nonlinear exterior problem, since we will move a little bit the points $\left(x_{i}\right)$.

\subsection{Harmonic extensions}

We study the properties of interior and exterior harmonic extensions. Given $\varphi \in \mathcal{C}^{2, \beta}\left(S^{1}\right)$ and define $H^{i}\left(=H^{i}(\phi ; \cdot)\right)$ to be the solution of

$$
\left\{\begin{aligned}
\Delta H^{i} & =0 \text { in } B_{1} \\
H^{i} & =\varphi \text { on } \partial B_{1}
\end{aligned}\right.
$$

We denote by $e_{1}, e_{2}$ the coordinate functions on $S^{1}$. 
Lemma 2 [21]If we assume that

$$
\int_{S^{1}} \varphi d v_{S^{1}}=0 \text { and } \int_{S^{1}} \varphi e_{\ell} d v_{S^{1}}=0 \text { for } \ell=1,2
$$

then there exists $c>0$ such that

$$
\left\|H^{i}(\varphi ; \cdot)\right\|_{\mathcal{C}_{2}^{2, \beta}\left(\bar{B}_{1}^{*}\right)} \leq \mathcal{C}\|\varphi\|_{\mathcal{C}^{2, \beta}\left(S^{1}\right)} .
$$

Given $\tilde{\varphi} \in \mathcal{C}^{2, \beta}\left(S^{1}\right)$, we define $H^{e}\left(=H^{e}(\tilde{\varphi} ; \cdot)\right)$ to be the solution of

$$
\left\{\begin{aligned}
\Delta H^{e} & =0 \text { in } \mathbb{R}^{2}-B_{1} \\
H^{e} & =\tilde{\varphi} \text { on } \partial B_{1}
\end{aligned}\right.
$$

which decays at infinity.

Definition 5 Given $k \in \mathbb{N}, \beta \in(0,1)$ and $v \in \mathbb{R}$, we define the space $\mathcal{C}_{v}^{k, \beta}\left(\mathbb{R}^{2}-B_{1}\right)$ as the space of functions $w \in \mathcal{C}_{\text {loc }}^{k, \beta}\left(\mathbb{R}^{2}-B_{1}\right)$ for which the following norm

$$
\|\left. w\right|_{\mathcal{C}_{v}^{k, \beta}\left(\mathbb{R}^{2}-B_{1}\right)}=\sup _{r \geq 1}\left(r^{-v}\|w(r \cdot)\|_{\mathcal{C}_{v}^{k, \beta}\left(\bar{B}_{2}-B_{1}\right)}\right),
$$

is finite.

Lemma 3 [21] If we assume that

$$
\int_{S^{1}} \tilde{\varphi} d v_{S^{1}}=0 .
$$

Then there exists $c>0$ such that

$$
\left\|H^{e}(\tilde{\varphi}, ; \cdot)\right\|_{\mathcal{C}_{-1}^{2, \beta}\left(\mathbb{R}^{2}-B_{1}\right)} \leq c\|\tilde{\varphi}\|_{\mathcal{C}^{2, \beta}\left(S^{1}\right)} .
$$

If $F \subset L^{2}\left(S^{1}\right)$ is a space of functions defined on $S^{1}$, we define the space $F_{\perp}$ to be the subspace of functions $F$ of which are $L^{2}\left(S^{1}\right)$-orthogonal to the functions $1, e_{1}, e_{2}$. We will need the:

Lemma 4 [21]The mapping

$$
\begin{array}{ccc}
\mathcal{P}: \quad \mathcal{C}^{2, \beta}\left(S^{1}\right)_{\perp} & \rightarrow & \mathcal{C}^{1, \beta}\left(S^{1}\right)_{\perp} \\
\psi & \mapsto & \partial_{r} H^{i}-\partial_{r} H^{e}
\end{array}
$$

where $H^{i}\left(=H^{i}(\psi ; \cdot)\right)$ and $H^{e}=H^{e}(\psi ; \cdot)$, is an isomorphism.

\section{The nonlinear interior problem}

We are interested in studying equations of type

$$
\Delta w+\lambda|\nabla w|^{2}+2\left(e^{w}+e^{\gamma w}\right)=0 .
$$

In $\bar{B}_{R_{\varepsilon, \lambda}}$.

Given $\varphi \in \mathcal{C}^{2, \beta}\left(S^{1}\right)$ satisfying (24), we define

$$
\mathbf{v}:=u_{1}+H^{i}\left(\varphi, \cdot / R_{\varepsilon, \lambda}\right)+h .
$$

Then, we look for a solution of (27) of the form $w=\mathbf{v}+v$ and using the fact that $H^{i}$ is harmonic, this amounts to solve 


$$
\begin{aligned}
\mathbb{L} v:=\mathfrak{S}(v)= & \frac{8}{\left(1+r^{2}\right)^{2}} e^{h}\left(e^{H^{i}\left(\varphi, \cdot / R_{\varepsilon, \lambda}\right)+v}-v-1\right)+\frac{8}{\left(1+r^{2}\right)^{2}}\left(e^{h}-1\right) v \\
& +\lambda\left|\nabla\left[u_{1}+H^{i}\left(\varphi, \cdot / R_{\varepsilon, \lambda}\right)+h+v\right]\right|^{2}-\lambda\left|\nabla\left(u_{1}+h\right)\right|^{2} \\
& +\frac{8 \varepsilon^{4(1-\gamma)}}{\left(\left(1+\varepsilon^{2}\right) \tau\right)^{2(1-\gamma)}\left(1+|x|^{2}\right)^{2 \gamma}} e^{\gamma h}\left(e^{\gamma H^{i}\left(\varphi, \cdot / R_{\varepsilon, \lambda}\right)+\gamma v}-1\right) .
\end{aligned}
$$

We fix $\mu \in(1,2)$ and denote by $\mathcal{G}_{\mu}$ to be a right inverse of $\mathbb{L}_{\mu}$ provided by Proposition 1 . To find a solution of (28), it is sufficient to find $v \in \mathcal{C}_{\mu}^{2, \beta}\left(\left(\mathbb{R}^{2}\right)\right)$ solution of

$$
v=\mathcal{G}_{\mu} \circ \mathcal{E}_{\mu} \circ \mathfrak{S}(v) .
$$

We denote by $\mathcal{N}\left(=\mathcal{N}_{\varepsilon, \tau, \varphi}\right)$, the nonlinear operator appearing on the right-hand side of equation (29).

Given $\kappa>0$ (whose value will be fixed later on), we further assume that the functions $\phi$ satisfy

$$
\|\varphi\|_{\mathcal{C}^{2, \beta}\left(S^{1}\right)} \leq \kappa r_{\varepsilon, \lambda}^{2} .
$$

Then, we have the following result

Lemma 5 Given $\kappa>0$. There exist $\varepsilon_{\kappa}>0, \lambda_{\kappa}>0, c_{\kappa}>0$ and $\bar{c}_{\kappa}>0$ (only depend on $\kappa)$ such that for all $\lambda \in\left(0, \lambda_{\kappa}\right)$ and $\varepsilon \in\left(0, \varepsilon_{\kappa}\right)$

$$
\|\mathcal{N}(0)\|_{\mathcal{C}_{\mu}^{2, \beta}\left(B_{R_{\varepsilon, \lambda}}\right)} \leq \mathcal{C}_{\kappa} r_{\varepsilon, \lambda}^{2} .
$$

and

$$
\left\|\mathcal{N}\left(v_{2}\right)-\mathcal{N}\left(v_{1}\right)\right\|_{\mathcal{C}_{\mu}^{2, \beta}\left(B_{R_{\varepsilon, \lambda}}\right)} \leq \mathcal{C}_{\kappa} r_{\varepsilon, \lambda}^{2}\left\|v_{2}-v_{1}\right\|_{\mathcal{C}_{\mu}^{2, \beta}\left(B_{R_{\varepsilon, \lambda}}\right)}
$$

provided $v_{1}, v_{2} \in \mathcal{C}_{\mu}^{2, \beta}\left(\mathbb{R}^{2}\right)$ satisfying $\left\|v_{i}\right\|_{\mathcal{C}_{\mu}^{2, \beta}\left(\mathbb{R}^{2}\right)} \leq 2 c_{\kappa} r_{\varepsilon, \lambda}^{2}$.

Proof. The proof of the first estimate follows from the asymptotic behavior of $H^{i}$ together with the assumption on the norm of boundary data $\phi$ given by (30). Indeed, let $c_{\kappa}$ be a constant depending only on $\kappa$ (provided $\varepsilon$ and $\lambda$ are chosen small enough) it follows from the estimate of $H^{i}$, given by lemma 2, that

$$
\left\|H^{i}\left(\cdot / R_{\varepsilon, \lambda}\right)\right\|_{\mathcal{C}_{2}^{2, \beta}\left(\bar{B}_{R_{\varepsilon, \lambda}}\right)} \leq c_{\kappa} R_{\varepsilon, \lambda}^{-2}\|\varphi\|_{\mathcal{C}^{2, \beta}} \leq c_{\kappa} \varepsilon^{2} .
$$

Since for each $x \in B_{R_{\varepsilon, \lambda}}$ we have

$$
|h(x)| \leq c_{\kappa} r_{\varepsilon, \lambda}^{2+\delta} \varepsilon^{-\delta} \leq\left\{\begin{array}{l}
\varepsilon^{(1+\gamma) / 2} \rightarrow 0 \text { as } \varepsilon \text { tends to } 0, \text { for } r_{\varepsilon, \lambda}=\sqrt{\varepsilon}, \\
\lambda^{1+\delta / 2} \varepsilon^{-\delta} \rightarrow 0 \text { as } \lambda \text { tends to } 0 \text { by }\left(A_{\lambda}\right), \text { for } r_{\varepsilon, \lambda}=\sqrt{\lambda} \\
\varepsilon^{2(1-\gamma)} \rightarrow 0 \text { as } \varepsilon \text { tends to } 0, \text { for } r_{\varepsilon, \lambda}=\varepsilon^{2(1-\gamma)-\delta / 2}
\end{array}\right.
$$

where $\delta \in(0,1-\gamma]$. Then

$$
\left\|\left(1+|\cdot|^{2}\right)^{-2} e^{h}\left(e^{H^{i}\left(\varphi \cdot / R_{\varepsilon, \lambda}\right)}-1\right)\right\|_{\mathcal{C}_{\mu-2}^{0, \beta}\left(\bar{B}_{R_{\varepsilon, \lambda}}\right)} \leq c_{\kappa} \varepsilon^{2} .
$$

On the other hand, using the condition $\left(A_{\lambda}\right)$, we have

$$
\begin{gathered}
\lambda \sup _{r \leq R_{\varepsilon, \lambda}} r^{2-\mu}\left|\nabla\left[u_{1}+H^{i}\left(\varphi, \cdot / R_{\varepsilon, \lambda}\right)+h\right]\right|^{2} \leq c_{\kappa} r_{\varepsilon, \lambda}^{2} \\
\lambda \sup _{r \leq R_{\varepsilon, \lambda}} r^{2-\mu}\left|\nabla\left[u_{1}+h\right]\right|^{2} \leq c_{\kappa} r_{\varepsilon, \lambda}^{2}
\end{gathered}
$$


and

$$
\begin{aligned}
& \left\|\frac{8 \varepsilon^{4(1-\gamma)}}{\left(\left(1+\varepsilon^{2}\right) \tau\right)^{2(1-\gamma)}\left(1+|x|^{2}\right)^{2 \gamma}} e^{\gamma h}\left(e^{\gamma H^{i}\left(\varphi, \cdot / R_{\varepsilon, \lambda}\right)}-1\right)\right\|_{\mathcal{C}_{\mu-2}^{0, \beta}\left(\bar{B}_{R_{\varepsilon, \lambda}}\right)} \\
& \leq c_{\kappa} \varepsilon^{2} \varepsilon^{4(1-\gamma)-\mu} \leq c_{\kappa} r_{\varepsilon, \lambda}^{2} .
\end{aligned}
$$

Making use of Proposition 1 together with (20), we get

$$
\|\mathcal{N}(0)\|_{\mathcal{C}_{\mu}^{2, \beta}\left(B_{R_{\varepsilon, \lambda}}\right)} \leq \mathcal{C}_{\kappa} r_{\varepsilon, \lambda}^{2}
$$

In order to derive the second estimate, we use the fact that, for $v_{1}, v_{2} \in \mathcal{C}_{\mu}^{2, \beta}\left(\mathbb{R}^{2}\right)$ satisfying $\left\|v_{i}\right\|_{\mathcal{C}_{\mu}^{2, \beta}\left(\mathbb{R}^{2}\right)} \leq 2 c_{\kappa} r_{\varepsilon, \lambda}^{2}$ for $i=1,2, \mu \in(1,2)$ and the condition $\left(A_{\lambda}\right)$, then there exist $c_{\kappa}>0$ (only depend on $\kappa$ ) such that

$$
\begin{aligned}
& \sup _{r \leq R_{\varepsilon, \lambda}} r^{2-\mu}\left|\mathfrak{S}\left(v_{2}\right)-\mathfrak{S}\left(v_{1}\right)\right| \\
& \leq \mathcal{C}_{\kappa} r_{\varepsilon, \lambda}^{2}\left\|v_{2}-v_{1}\right\|_{\mathcal{C}_{\mu}^{2, \beta}\left(\mathbb{R}^{2}\right)}+c_{\kappa} \lambda\left\|v_{2}-v_{1}\right\|_{\mathcal{C}_{\mu}^{2, \beta}\left(\mathbb{R}^{2}\right)}+c_{\kappa} r_{\varepsilon, \lambda}^{2}\left\|v_{2}-v_{1}\right\|_{\mathcal{C}_{\mu}^{2, \beta}\left(\mathbb{R}^{2}\right)} .
\end{aligned}
$$

Similarly, making use of Proposition 1 together with (19), we conclude that there exists $\bar{c}_{\kappa}>0$ (only depend on $\kappa$ ) such that

$$
\left\|\mathcal{N}\left(v_{2}\right)-\mathcal{N}\left(v_{1}\right)\right\|_{\mathcal{C}_{\mu}^{2, \beta}\left(B_{R_{\varepsilon, \lambda}}\right)} \leq \bar{c}_{\kappa} r_{\varepsilon, \lambda}^{2}\left\|v_{2}-v_{1}\right\|_{\mathcal{C}_{\mu}^{2, \beta}\left(B_{R_{\varepsilon, \lambda}}\right)}
$$

Reducing $\lambda_{\kappa}>0$ and $\varepsilon_{\kappa}>0$ if necessary, we can assume that, $\bar{c}_{\kappa} r_{\varepsilon, \lambda}^{2} \leq \frac{1}{2}$ for all $\lambda \in$ $\left(0, \lambda_{\kappa}\right)$ and $\varepsilon \in\left(0, \varepsilon_{\kappa}\right)$. Then, (31) and (32) are enough to show that $v \mapsto \mathcal{N}(v)$ is a contraction from $\left\{v \in \mathcal{C}_{\mu}^{2, \beta}\left(\mathbb{R}^{2}\right):\|v\|_{\mathcal{C}_{\mu}^{2, \beta}\left(\mathbb{R}^{2}\right)} \leq 2 c_{\kappa} r_{\varepsilon, \lambda}^{2}\right\}$ into itself and hence has a unique fixed point $v\left(=\bar{v}_{\varepsilon, \tau, \varphi}\right)$ in this set. This fixed point is solution of (20) in $\mathbb{R}^{2}$. We summarize this in the following:

Proposition 4 Given $\kappa>0$, there exist $\varepsilon_{\kappa}>0, \lambda_{\kappa}>0$ and $c_{\kappa}>0$ (only depending on $\kappa$ ) such that for all $\varepsilon \in\left(0, \varepsilon_{\kappa}\right), \lambda \in\left(0, \lambda_{\kappa}\right)$ satisfying $(A)$, for all $\tau$ in some fixed compact subset of $\left[\tau-, \tau^{+}\right] \subset(0, \infty)$ and for a given $\phi$ satisfying (24)-(30), then there exists a unique $v\left(:=\bar{v}_{\varepsilon, \tau, \varphi}\right)$ solution of (29) such that

$$
w:=u_{1}+H^{i}\left(\varphi, \cdot / R_{\varepsilon, \lambda}\right)+h+\bar{v}_{\varepsilon, \tau, \varphi}
$$

Solve (27) in $\bar{B}_{R_{\varepsilon, \lambda}}$. In addition,

$$
\|v\|_{\mathcal{C}_{\mu}^{2, \beta}\left(\mathbb{R}^{2}\right)} \leq 2 c_{\kappa} r_{\varepsilon, \lambda}^{2}
$$

Observe that the function $v\left(:=\bar{v}_{\varepsilon, \tau, \varphi}\right)$ being obtained as a fixed point for contraction mappings, it depends continuously on the parameter $\tau$.

\section{The nonlinear exterior problem}

Recall that $G(\cdot, \tilde{x})$ denote the unique solution of

$$
-\Delta G(\cdot, \tilde{x})=8 \pi \delta_{\tilde{x}}
$$

in $\Omega$, with $G(\cdot, \tilde{x})=0$ on $\partial \Omega$. In addition, the following decomposition holds

$$
G(x, \tilde{x})=-4 \log |x-\tilde{x}|+H(x, \tilde{x})
$$


where $x \mapsto H(x, \tilde{x})$ is a smooth function. Here, we give an estimate of the gradient of $H(x, \tilde{x})$ without proof (see [14], Lemma 2.1), there exists a constant $c>0$, so that

$$
\|\nabla H(\cdot, \tilde{x})\|_{\infty} \leq c d(\tilde{x}, \partial \Omega)^{-1} .
$$

Let $\tilde{\mathbf{x}}:=\left(\tilde{x}_{1}, \ldots, \tilde{x}_{m}\right)$ close enough to $\mathbf{x}:=\left(x_{1}, \ldots, x_{m}\right), \tilde{\eta}:=\left(\tilde{\eta}^{1}, \ldots, \tilde{\eta}^{m}\right) \in \mathbb{R}^{m}$ close to 0 and $\tilde{\varphi}:=\left(\tilde{\varphi}^{1}, \ldots, \tilde{\varphi}^{m}\right) \in\left(\mathcal{C}^{2, \beta}\left(S^{1}\right)\right)^{m}$ satisfying (26). We define

$$
\tilde{\mathbf{v}}:=\sum_{i=1}^{m}\left(1+\tilde{\eta}^{i}\right) G\left(\cdot, \tilde{x}_{i}\right)+\sum_{i=1}^{m} \chi_{r_{0}}\left(\cdot-\tilde{x}_{i}\right) H^{e}\left(\tilde{\varphi}^{i} ;\left(\cdot-\tilde{x}_{i}\right) / r_{\varepsilon, \lambda}\right) .
$$

where $\chi_{r_{0}}$ is a cutoff function identically equal to 1 in $B_{r_{0} / 2}$ and identically equal to 0 outside $B_{r_{0}}$

We would like to find a solution of

$$
\Delta v+\lambda|\nabla v|^{2}+\rho^{2}\left(e^{v}+e^{\gamma v}\right)=0
$$

in $\bar{\Omega}_{r_{\varepsilon, \lambda}}(\tilde{\mathbf{x}}):=\bar{\Omega}-\cup_{i=1}^{m} B_{r_{\varepsilon, \lambda}}\left(\tilde{x}_{i}\right)$ which is a perturbation of $\tilde{\mathbf{v}}$. Writing $v=\tilde{\mathbf{v}}+\tilde{v}$. This amounts to solve

$$
-\Delta \tilde{v}=\rho^{2}\left(e^{\tilde{\mathbf{v}}+\tilde{v}}+e^{\gamma \tilde{\mathbf{v}}+\gamma \tilde{v}}\right)+\lambda|\nabla(\tilde{\mathbf{v}}+\tilde{v})|^{2}+\Delta \tilde{\mathbf{v}} .
$$

We need to define some auxiliary weighted spaces:

Definition 6 Let $\bar{r} \in\left(0, r_{0} / 2\right), k \in \mathbb{R}, \beta \in(0,1)$ and $v \in \mathbb{R}$, we define the Hölder weighted space $\mathcal{C}_{v}^{k, \beta}\left(\bar{\Omega}_{\bar{r}}(\mathbf{x})\right)$ as the set of functions $w \in \mathcal{C}^{k, \beta}\left(\bar{\Omega}_{\bar{r}}(\mathbf{x})\right)$ for which the following norm

$$
\left\|\left.w\right|_{\mathcal{C}_{v}^{k_{v}, \beta}\left(\bar{\Omega}_{\bar{\tau}}(\mathrm{x})\right)}:=\right\| w \|_{\mathcal{C}^{k, \beta}\left(\bar{\Omega}_{r_{0} / 2}(\mathrm{x})\right)}+\sum_{i=1}^{m} \sup _{r \in\left[\bar{r}, r_{0} / 2\right)}\left(r^{-v}\left\|w\left(x_{i}+r \cdot\right)\right\|_{\mathcal{C}^{k, \beta}\left(\bar{B}_{2}-B_{1}\right)}\right) .
$$

is finite

For all $\sigma \in\left(0, r_{0} / 2\right)$ and all $Y=\left(y_{1}, \ldots, y_{m}\right) \in \Omega^{m}$ such that $\|X-Y\| \leq r_{0} / 2$, where $X$ $=\left(x_{1}, \ldots, x_{m}\right)$, we denote by

$$
\tilde{\mathcal{E}}_{\sigma, Y}: \mathcal{C}_{v}^{0, \beta}\left(\bar{\Omega}_{\sigma}(Y)\right) \rightarrow \mathcal{C}_{v}^{0, \beta}\left(\bar{\Omega}^{*}(Y)\right),
$$

the extension operator defined by $\tilde{\mathcal{E}}_{\sigma, Y}(f)=f$ in $\bar{\Omega}_{\sigma}(Y)$

$$
\tilde{\mathcal{E}}_{\sigma, Y}(f)\left(y_{i}+x\right)=\tilde{\chi}\left(\frac{|x|}{\sigma}\right) f\left(y_{i}+\sigma \frac{x}{|x|}\right)
$$

for each $i=1, \ldots, m$ and $\tilde{\mathcal{E}}_{\sigma, Y}(f)=0$ in each $B_{\sigma / 2}\left(y_{i}\right)$, where $t \mapsto \tilde{\chi}(t)$ is a cutoff function identically equal to 1 for $t \geq 1$ and identically equal to 0 for $t \leq 1 / 2$. It is easy to check that there exists a constant $c=c(v)>0$ only depending on $v$ such that

$$
\left\|\tilde{\mathcal{E}}_{\sigma, Y}(w)\right\|_{\mathcal{C}_{v}^{0, \beta}\left(\bar{\Omega}^{*}(Y)\right)} \leq c\|w\|_{\mathcal{C}_{v}^{0, \beta}\left(\bar{\Omega}_{\sigma}(Y)\right)} .
$$

We fix

$$
v \in(-1,0)
$$

and denote by $\tilde{\mathcal{G}}_{v}: \mathcal{C}_{\nu-2}^{0, \beta}\left(\bar{\Omega}^{*}(\tilde{\mathbf{x}})\right) \rightarrow \mathcal{C}_{v}^{2, \beta}\left(\bar{\Omega}^{*}(\tilde{\mathbf{x}})\right)$ a right inverse of $\Delta$ provided by Proposition 3 with $\bar{\Omega}^{*}(\tilde{\mathbf{x}})=\bar{\Omega}-\left\{\tilde{x}_{1}, \ldots, \tilde{x}_{m}\right\}$. Clearly, it is enough to find $\tilde{v} \in \mathcal{C}_{v}^{2, \beta}\left(\bar{\Omega}^{*}(\tilde{\mathbf{x}})\right)$ 
solution of

$$
\tilde{v}=\tilde{\mathcal{G}}_{\nu} \circ \tilde{\mathcal{E}}_{r_{\varepsilon, \lambda}, \tilde{\mathbf{x}}}\left(\rho^{2}\left(e^{\tilde{\mathbf{v}}+\tilde{v}}+e^{\gamma \tilde{\mathbf{v}}+\gamma \tilde{v}}\right)+\lambda|\nabla(\tilde{\mathbf{v}}+\tilde{v})|^{2}+\Delta \tilde{\mathbf{v}}\right)=\tilde{\mathcal{G}}_{\nu} \circ \tilde{\mathcal{E}}_{r_{\varepsilon, \lambda}, \tilde{\mathbf{x}}} \circ \tilde{\mathfrak{R}}(\tilde{v}) .
$$

where

$$
\tilde{\mathfrak{R}}(\tilde{v})=\rho^{2}\left(e^{\tilde{\mathbf{v}} \tilde{v}}+e^{\gamma \tilde{\mathbf{v}}+\gamma \tilde{v}}\right)+\lambda|\nabla(\tilde{\mathbf{v}}+\tilde{v})|^{2}+\Delta \tilde{\mathbf{v}} .
$$

We denote by $\tilde{\mathcal{N}}\left(=\tilde{\mathcal{N}}_{\varepsilon, \tilde{r}, \tilde{\mathbf{x}}, \tilde{\varphi})}\right.$ the nonlinear operator which appears on the right hand side of Eq.(36). Given $\kappa>0$ (whose value will be fixed later on), we assume that the points $\tilde{\mathbf{x}}=\left(\tilde{x}_{1}, \ldots, \tilde{x}_{m}\right)$, the functions $\tilde{\varphi}=\left(\tilde{\varphi}^{1}, \ldots, \tilde{\varphi}^{m}\right)$ and the parameters $\tilde{\eta}=\left(\tilde{\eta}^{1}, \ldots, \tilde{\eta}^{m}\right)$ to satisfy

$$
\begin{aligned}
& \left|\tilde{x}_{i}-x_{i}\right| \leq \kappa r_{\varepsilon, \lambda} \\
& \left\|\tilde{\varphi}^{i}\right\|_{\mathcal{C}^{2, \beta}\left(S^{1}\right)} \leq \kappa r_{\varepsilon, \lambda}^{2}
\end{aligned}
$$

and

$$
\left|\tilde{\eta}^{i}\right| \leq \kappa r_{\varepsilon, \lambda}^{2}
$$

Then, the following result holds

Lemma 6 Given $\kappa>0$, there exist $\varepsilon_{\kappa}>0, \lambda_{\kappa}>0, c_{\kappa}>0$ and $\bar{c}_{\kappa}>0$ (depending on $\kappa$ ) such that for all $\varepsilon \in\left(0, \varepsilon_{\kappa}\right), \lambda \in\left(0, \lambda_{\kappa}\right)$

$$
\|\tilde{\mathcal{N}}(0)\|_{\mathcal{C}_{v}^{2, \beta}\left(\bar{\Omega}^{*}(\tilde{\mathbf{x}})\right)} \leq c_{\kappa} r_{\varepsilon, \lambda}^{2}
$$

and

$$
\left\|\tilde{\mathcal{N}}\left(\tilde{v}_{2}\right)-\tilde{\mathcal{N}}\left(\tilde{v}_{1}\right)\right\|_{\mathcal{C}_{v}^{2, \beta}\left(\bar{\Omega}^{*}(\tilde{x})\right)} \leq c_{\kappa} r_{\varepsilon, \lambda}^{2}\left\|\tilde{v}_{2}-\tilde{v}_{1}\right\|_{\mathcal{C}_{v}^{2, \beta}\left(\bar{\Omega}^{*}(\tilde{\mathbf{x}})\right)}
$$

provided $\tilde{v}_{1}, \tilde{v}_{2} \in \mathcal{C}_{v}^{2, \beta}\left(\bar{\Omega}^{*}(\tilde{\mathbf{x}})\right)$ and satisfy $\left\|\tilde{v}_{i}\right\|_{\mathcal{C}_{v}^{2, \beta}\left(\bar{\Omega}^{*}(\tilde{\mathbf{x}})\right)} \leq 2 c_{\kappa} r_{\varepsilon, \lambda}^{2}$.

Proof: Recall that $\tilde{\mathcal{N}}(\tilde{v})=\tilde{\mathcal{G}}_{\nu} \circ \tilde{\Re}(\tilde{v})$, we will estimate $\tilde{\mathcal{N}}(0)$ in different subregions of $\bar{\Omega}^{*}(\tilde{\mathbf{x}})$.

*In $B_{r_{0}}\left(\tilde{x}_{i}\right)$, we have $\chi_{r_{0}}\left(x-\tilde{x}_{i}\right)=1, \Delta \tilde{\mathbf{v}}=0$ and

$$
\left|H^{e}\left(\tilde{\varphi}_{i} ;\left(x-\tilde{x}_{i}\right) / r_{\varepsilon, \lambda}\right)\right| \leq \kappa r_{\varepsilon, \lambda}^{3} r^{-1}
$$

so that

$$
\begin{aligned}
|\tilde{\mathfrak{R}}(0)| \leq & c_{\kappa} \varepsilon^{2}\left|x-\tilde{x}_{i}\right|^{-4\left(1+\tilde{\eta}^{i}\right)} \prod_{\ell=1, \ell \neq i}^{m}\left|x-\tilde{x}_{\ell}\right|^{-4\left(1+\tilde{\eta}^{\ell}\right)} \\
& +\varepsilon^{2}\left|x-\tilde{x}_{i}\right|^{-4 \gamma\left(1+\tilde{\eta}^{i}\right)} \prod_{\ell=1, \ell \neq i}^{m}\left|x-\tilde{x}_{\ell}\right|^{-4 \gamma\left(1+\tilde{\eta}^{\ell}\right)}+\lambda|\nabla \tilde{\mathbf{v}}|^{2} \\
\leq & c_{\kappa} \varepsilon^{2} r^{-4\left(1+\tilde{\eta}^{i}\right)}+\left.\lambda\left|\left(1+\tilde{\eta}^{i}\right) r^{-1}+\left(1+\tilde{\eta}^{i}\right)\right| \nabla H(x, \tilde{x})|+| \nabla H^{e}\left(\tilde{\varphi}^{i} ;\left(\cdot-\tilde{x}_{i}\right) / r_{\varepsilon, \lambda}\right)\right|^{2} \\
\leq & c_{\kappa} \varepsilon^{2} r^{-4\left(1+\tilde{\eta}^{i}\right)}+c_{\kappa} \lambda\left(\left(1+\tilde{\eta}^{i}\right) r^{-1}+\left(1+\tilde{\eta}^{i}\right) \log r+r_{\varepsilon, \lambda}^{2} r^{-2}\right)^{2} .
\end{aligned}
$$

Hence, for $v \in(-1,0)$ and for $\tilde{\eta}^{i}$ small enough, we get

$$
\|\tilde{\mathfrak{R}}(0)\|_{\mathcal{C}_{v-2}^{0, \beta}\left(\cup_{i=1}^{m} B_{r_{0}}\left(\tilde{x}_{i}\right)\right)} \leq \sup _{r_{\varepsilon, \lambda} \leq r \leq r_{0} / 2} r^{2-\nu}|\tilde{\Re}(0)| \leq c_{\kappa} \varepsilon^{2} r_{\varepsilon, \lambda}^{-2}+c_{\kappa} \lambda .
$$


* In $\Omega-B_{r_{0}}\left(\tilde{x}_{i}\right)$, we have $\chi_{r_{0}}\left(x-\tilde{x}_{i}\right)=1$ and $\Delta \tilde{\mathbf{v}}=0$. Thus

$$
|\tilde{\Re}(0)| \leq c_{\kappa} \varepsilon^{2} \prod_{\ell=1}^{m} e^{\left(1+\tilde{\eta}^{\ell}\right) G\left(x, \tilde{x}_{\ell}\right)}+c_{\kappa} \lambda\left(\left(1+\tilde{\eta}^{i}\right) r^{-1}+\left(1+\tilde{\eta}^{i}\right) \log r+r_{\varepsilon, \lambda}^{2} r^{-2}\right)^{2} .
$$

So, for $v \in(-1,0)$, we have

$$
\|\left.\tilde{\mathfrak{R}}(0)\right|_{\mathcal{C}_{v-2}^{0, \beta}\left(\Omega-\cup_{i=1}^{m} B_{r_{0}}\left(\tilde{x}_{i}\right)\right)} \leq \sup _{r_{0} \leq r} r^{2-v}|\tilde{\mathfrak{R}}(0)| \leq c_{\kappa} \varepsilon^{2}+c_{\kappa} \lambda .
$$

* In $B_{r_{0}}\left(\tilde{x}_{i}\right)-B_{r_{0} / 2}\left(\tilde{x}_{i}\right)$, using the estimat (40), then we have

$$
\begin{aligned}
|\tilde{\mathfrak{R}}(0)| \leq & \left.c_{\kappa} \varepsilon^{2} r^{-4\left(1+\tilde{\eta}^{i}\right.}\right)+c_{\kappa} \lambda\left(\left(1+\tilde{\eta}^{i}\right) r^{-1}+\left(1+\tilde{\eta}^{i}\right) \log r+r_{\varepsilon, \lambda}^{2} r^{-2}\right)^{2} \\
& +\sum_{i=1}^{m}\left|\left[\Delta, \chi_{r_{0}}\left(x-\tilde{x}_{i}\right)\right]\right|\left|H^{e}\left(\tilde{\varphi}^{i} ;\left(x-\tilde{x}_{i}\right) / r_{\varepsilon, \lambda}\right)\right| \\
\leq & c_{\kappa}\left(\varepsilon^{2}+c_{\kappa} \lambda\left(\left(1+\tilde{\eta}^{i}\right) r^{-1}+\left(1+\tilde{\eta}^{i}\right) \log r+r_{\varepsilon, \lambda}^{2} r^{-2}\right)^{2}+r^{-1} r_{\varepsilon, \lambda}^{3}\right),
\end{aligned}
$$

where

$$
\left[\Delta, \chi_{r_{0}}\right] w=\Delta w \chi_{r_{0}}+w \Delta \chi_{r_{0}}+2 \nabla w \cdot \nabla \chi_{r_{0}} .
$$

Then

$$
\|\left.\tilde{\mathfrak{R}}(0)\right|_{\mathcal{C}_{v-2}^{0, \beta}\left(\cup_{i=1}^{m}\left(B_{r_{0}}\left(\tilde{x}_{i}\right)-B_{r_{0} / 2}\left(\tilde{x}_{i}\right)\right)\right)} \leq \sup _{r_{0} / 2 \leq r \leq r_{0}} r^{2-v}|\tilde{\Re}(0)| \leq c_{\kappa} r_{\varepsilon, \lambda}^{2}+c_{\kappa} \lambda .
$$

So,

$$
\|\tilde{\Re}(0)\|_{\mathcal{C}_{v-2}^{0, \beta}\left(\cup_{i=1}^{m}\left(\Omega-B_{r_{0}}\left(\tilde{x}_{i}\right)\right)\right.} \leq c_{\kappa} r_{\varepsilon, \lambda}^{2} .
$$

Making use of Proposition 3 together with (34), we conclude that

$$
\|\tilde{\mathcal{N}}(0)\|_{\mathcal{C}_{v}^{2, \beta}\left(\bar{\Omega}^{*}(\mathrm{x})\right)} \leq c_{\kappa} r_{\varepsilon, \lambda}^{2}
$$

For the proof of the second estimate, let $\tilde{v}_{1}$ and $\tilde{v}_{2} \in C_{\nu}^{2, \beta}\left(\bar{\Omega}^{*}(\tilde{\mathbf{x}})\right)$ satisfying $\left\|\tilde{v}_{i}\right\|_{\mathcal{C}_{\mu}^{2, \beta}\left(\bar{\Omega}^{*}(\tilde{x})\right)} \leq 2 c_{\kappa} r_{\varepsilon, \lambda}^{2}$ for $i=1,2$, we have

$$
\left|\tilde{\mathfrak{R}}_{(}\left(\tilde{v}_{2}\right)-\tilde{\mathfrak{R}}\left(\tilde{v}_{1}\right)\right| \leq c_{\kappa} \varepsilon^{2} e^{\tilde{\mathbf{v}}}\left|e^{\tilde{\nu}_{2}}-e^{\tilde{e}_{1}}\right|+c_{\kappa} \varepsilon^{2} e^{\gamma \tilde{\mathbf{v}}}\left|e^{\gamma \tilde{v}_{2}}-e^{\gamma \tilde{v}_{1}}\right|+\left.\lambda|| \nabla\left(\tilde{\mathbf{v}}+\tilde{v}_{2}\right)\right|^{2}-\left|\nabla\left(\tilde{\mathbf{v}}+\tilde{v}_{1}\right)\right|^{2} \mid .
$$

Then for $\gamma \in(0,1)$, we get

$$
\begin{aligned}
\left|\tilde{\Re}_{i}\left(\tilde{v}_{2}\right)-\tilde{\mathfrak{R}}\left(\tilde{v}_{1}\right)\right| & \leq c_{\kappa} \varepsilon^{2}\left|x-x_{i}\right|^{-4\left(1+\tilde{\eta}^{i}\right)}\left|\tilde{v}_{2}-\tilde{v}_{1}\right|+c_{\kappa} \lambda\left|\nabla\left(\tilde{v}_{2}-\tilde{v}_{1}\right)\right|\left(\left|\nabla\left(\tilde{v}_{2}+\tilde{v}_{1}\right)\right|+2|\nabla \tilde{\mathbf{v}}|\right) \\
& \leq c_{\kappa} \varepsilon^{2} r^{-4\left(1+\tilde{\eta}_{i j}\right)}\left|\tilde{v}_{2}-\tilde{v}_{1}\right|+c_{\kappa} \lambda\left|\nabla\left(\tilde{v}_{2}-\tilde{v}_{1}\right)\right|\left(\left|\nabla\left(\tilde{v}_{2}+\tilde{v}_{1}\right)\right|+2|\nabla \tilde{\mathbf{v}}|\right) .
\end{aligned}
$$

So, for $\tilde{\eta}^{i}$ small enough and using the estimate (35), there exist $\bar{c}_{\kappa}$ (depending on $\kappa$ ), such that:

$$
\left\|\tilde{\mathcal{N}}\left(\tilde{v}_{2}\right)-\tilde{\mathcal{N}}\left(\tilde{v}_{1}\right)\right\|_{\mathcal{C}_{v}^{2, \beta}\left(\bar{\Omega}^{*}(\tilde{\mathbf{x}})\right)} \leq c_{\kappa} r_{\varepsilon, \lambda}^{2}\left\|\tilde{v}_{2}-\tilde{v}_{1}\right\|_{\mathcal{C}_{v}^{2, \beta}\left(\bar{\Omega}^{*}(\tilde{\mathbf{x}})\right)} .
$$

口

Reducing $\lambda_{\kappa}>0$ and $\varepsilon_{\kappa}>0$ if necessary, we can assume that, $\bar{c}_{\kappa} r_{\varepsilon, \lambda}^{2} \leq \frac{1}{2}$ for all $\lambda \in$ $\left(0, \lambda_{\kappa}\right)$ and $\varepsilon \in\left(0, \varepsilon_{\kappa}\right)$. Then, (42) and (43) are enough to show that $\tilde{v} \mapsto \tilde{\mathcal{N}}(\tilde{v})$ is a contraction from $\left\{\tilde{v} \in \mathcal{C}_{v}^{2, \beta}\left(\mathbb{R}^{2}\right):\|\tilde{v}\|_{\mathcal{C}_{v}^{2, \beta}\left(\mathbb{R}^{2}\right)} \leq 2 c_{\kappa} r_{\varepsilon, \lambda}^{2}\right\}$ into itself and hence has a 
unique fixed point $\tilde{v}\left(=\bar{v}_{\varepsilon, \tau, \varphi}\right)$ in this set. This fixed point is solution of (35). We summarize this in the following

Proposition 5 Given $\kappa>0$, there exists $\varepsilon_{\kappa}>0$ and $\lambda_{\kappa}>0$ (depending on $\kappa$ ) such that for all $\varepsilon \in\left(0, \varepsilon_{\kappa}\right)$ and $\lambda \in\left(0, \lambda_{\kappa}\right)$, for all set of parameter $\tilde{\eta}^{i}$ satisfying (39) and function

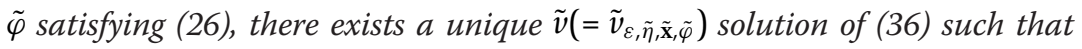

$$
\|\tilde{v}\|_{\mathcal{C}_{v}^{2, \beta}\left(\bar{\Omega}^{*}(\mathrm{x})\right)} \leq 2 \bar{c}_{\kappa} r_{\varepsilon, \lambda}^{2} .
$$

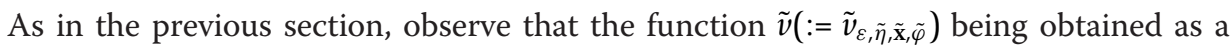
fixed point for contraction mapping, depends smoothly on the parameters $\tilde{\eta}$ and the points $\tilde{\mathbf{x}}$.

\section{The nonlinear Cauchy-data matching}

Keeping the notations of the previous sections, we gather the results of Proposition 4 and 5. Assume that $\tilde{\mathbf{x}}:=\left(\tilde{x}_{1}, \ldots, \tilde{x}_{m}\right) \in \Omega^{m} \in \Omega^{m}$ are given close to $\mathbf{x}:=\left(x_{1}, \ldots, x_{m}\right)$ and satisfy (37). Assume also that $\tau:=\left(\tau_{1}, \ldots, \tau_{m}\right) \in\left[\tau_{-}, \tau^{+}\right]^{m} \subset(0, \infty)^{m}$ are given (the values of $\tau_{-}$and $\tau^{+}$will be fixed shortly). First, we consider some set of boundary data $\varphi:=\left(\varphi^{1}, \ldots, \varphi^{m}\right) \in\left(\mathcal{C}^{2, \beta}\left(S^{1}\right)\right)^{m}$ satisfying (24). We set

$$
R_{\varepsilon, \lambda}^{i}=\tau_{i} r_{\varepsilon, \lambda} / \varepsilon \text {. }
$$

According to the result of Proposition 4, we can find $v_{i n t}^{i}$ a solution of

$$
\Delta v+\lambda|\nabla v|^{2}+\rho^{2}\left(e^{v}+e^{\gamma v}\right)=0
$$

in each $B_{r_{\varepsilon, \lambda}}\left(\tilde{x}_{i}\right)$ that can be decomposed as

$$
v_{\text {int }}^{i}(x)=v_{\varepsilon, \tau_{i}}\left(x-\tilde{x}_{i}\right)+h\left(R_{\varepsilon, \lambda}^{i}\left(x-\tilde{x}_{i}\right) / r_{\varepsilon, \lambda}\right)+H^{i}\left(\varphi^{i} ;\left(x-\tilde{x}_{i}\right) / r_{\varepsilon, \lambda}\right)+\bar{v}_{\varepsilon, \tau_{i}, \varphi^{i}}\left(R_{\varepsilon, \lambda}^{i}\left(x-\tilde{x}_{i}\right) / r_{\varepsilon, \lambda}\right),
$$

where the function $v^{i}=\bar{v}_{\varepsilon, \tau_{i}, \varphi^{i}}$ satisfies

$$
\left\|v^{i}\right\|_{\mathcal{C}_{\mu}^{2, \beta}\left(\mathbb{R}^{2}\right)} \leq 2 c_{\kappa} r_{\varepsilon, \lambda}^{2} .
$$

Similarly, given some boundary data $\tilde{\varphi}=\left(\tilde{\varphi}^{1}, \ldots, \tilde{\varphi}^{m}\right) \in\left(\mathcal{C}^{2, \beta}\left(S^{1}\right)\right)^{m}$ satisfying (26), some parameters $\tilde{\eta}:=\left(\tilde{\eta}^{1}, \ldots, \tilde{\eta}^{m}\right) \in \mathbb{R}^{m}$ satisfying (38), provide $\varepsilon \in\left(0, \varepsilon_{\kappa}\right)$ and $\lambda \in(0$, $\lambda_{\kappa}$ ), we use the result of Proposition 5, to find a solution $v_{\text {ext }}$ of (43) which can be decomposed as

$$
v_{\text {ext }}=\sum_{i=1}^{m}\left(1+\tilde{\eta}^{i}\right) G\left(\cdot, \tilde{x}_{i}\right)+\sum_{i=1}^{m} \chi_{r_{0}}\left(\cdot-\tilde{x}_{i}\right) H^{e}\left(\tilde{\varphi}^{i} ;\left(\cdot-\tilde{x}_{i}\right) / r_{\varepsilon, \lambda}\right)+\tilde{v}_{\varepsilon, \tilde{\eta}, \tilde{\mathbf{x}}, \tilde{\varphi}}
$$

in $\bar{\Omega}_{r_{\varepsilon, \lambda}}$ where, the function $\tilde{v}\left(:=\tilde{v}_{\varepsilon, \tilde{\eta}, \tilde{\mathbf{x}}, \tilde{\varphi}}\right) \in \mathcal{C}_{\nu}^{2, \beta}\left(\bar{\Omega}^{*}(\tilde{\mathbf{x}})\right)$ satisfies

$$
\|\tilde{v}\|_{\mathcal{C}_{v}^{2, \beta}\left(\bar{\Omega}^{*}(\tilde{\mathbf{x}})\right)} \leq 2 c_{\kappa} r_{\varepsilon, \lambda}^{2} .
$$

It remains to determine the parameters and the functions in such a way that the function which is equal to $v_{\text {int }}^{i}$ in $\cup_{i=1}^{m} B_{r_{\varepsilon, \lambda}}\left(\tilde{x}_{i}\right)$ and that is equal to $v_{\text {ext }}$ in $\bar{\Omega}_{r_{\varepsilon, \lambda}}(\tilde{\mathbf{x}})$ ) is a smooth function. This amounts to find the boundary data and the parameters so that, for each $i=1 \ldots, m$

$$
v_{\mathrm{int}}^{i}=v_{\mathrm{ext}} \quad \text { and } \quad \partial_{r} v_{\mathrm{int}}^{i}=\partial_{r} v_{\mathrm{ext}},
$$


on $\partial B_{r_{\varepsilon, \lambda}}\left(\tilde{x}_{i}\right)$. Assuming we have already done so, this provides for each $\varepsilon$ and $\lambda$ small enough a function $v_{\varepsilon, \lambda} \in \mathcal{C}^{2, \beta}$ (which is obtained by patching together the functions $v_{\text {int }}^{i}$ and the function $\left.v_{\text {ext }}\right)$ solution of $-\Delta v-\lambda|\nabla v|^{2}=\rho^{2}\left(e^{v}+e^{\gamma v}\right)$ and elliptic regularity theory implies that this solution is in fact smooth. This will complete the proof of our result since, as $\varepsilon$ and $\lambda$ tend to 0 , the sequence of solutions we have obtained satisfies the required properties, namely, away from the points $x_{i}$ the sequence $v_{\varepsilon, \lambda}$ converges to $\sum_{i} G\left(\cdot, x_{i}\right)$. Before we proceed, the following remarks are due. First, it will be convenient to observe that the function $v_{\varepsilon, \tau_{i}}$ can be expanded as

$$
v_{\varepsilon, \tau_{i}}(x)=-2 \log \tau_{i}-4 \log |x|+\mathcal{O}\left(\frac{\varepsilon^{2} \tau_{i}^{-2}}{|x|^{2}}\right)
$$

near $\partial B_{r_{\varepsilon, \lambda}}$ The function

$$
\sum_{\ell=1}^{m}\left(1+\tilde{\eta}^{\ell}\right) G\left(x, \tilde{x}_{\ell}\right)
$$

which appear in the expression of $v_{\text {ext }}$ can be expanded as

$$
\sum_{\ell=1}^{m}\left(1+\tilde{\eta}^{\ell}\right) G\left(x+\tilde{x}_{i}, \tilde{x}_{\ell}\right)=-4\left(1+\tilde{\eta}^{i}\right) \log |x|+\mathcal{F}_{i}\left(\tilde{\mathbf{x}} ; \tilde{x}_{i}\right)+\nabla \mathcal{F}_{i}\left(\tilde{\mathbf{x}} ; \tilde{x}_{i}\right) \cdot x+\mathcal{O}\left(r_{\varepsilon, \lambda}^{2}\right)
$$

Near $\partial B_{r_{\varepsilon, \lambda}}\left(\tilde{x}_{i}\right)$. Here, we have defined

$$
\mathcal{F}_{i}\left(\tilde{\mathbf{x}}_{i} \cdot\right):=H\left(\tilde{x}_{i}, \cdot\right)+\sum_{\ell \neq i} G\left(\tilde{x}_{\ell} \cdot \cdot\right) .
$$

Thus for $x$ near $\partial B_{r_{\varepsilon, \lambda}}$, we have

$$
\begin{aligned}
\left(v_{\text {int }}^{i}-v_{\text {ext }}\right)(x)= & -2 \log \tau_{i}+4 \tilde{\eta}^{i} \log \left|x-\tilde{x}_{i}\right|+h\left(R_{\varepsilon, \lambda}^{i}\left(x-\tilde{x}_{i}\right) / r_{\varepsilon, \lambda}\right) \\
& +H^{i}\left(\varphi^{i} ;\left(x-\tilde{x}_{i}\right) / r_{\varepsilon, \lambda}\right)-H^{e}\left(\tilde{\varphi}^{i} ;\left(x-\tilde{x}_{i}\right) / r_{\varepsilon, \lambda}\right) \\
& -\left(\left(1+\tilde{\eta}^{i}\right) H\left(x, \tilde{x}_{i}\right)+\sum_{\ell=1, \ell \neq i}^{m}\left(1+\tilde{\eta}^{\ell}\right) G\left(x, \tilde{x}_{\ell}\right)\right)+\mathcal{O}\left(\frac{\varepsilon^{2} \tau_{i}^{-2}}{\left|x-\tilde{x}_{i}\right|^{2}}\right)+\mathcal{O}\left(r_{\varepsilon, \lambda}^{2}\right) \\
= & -2 \log \tau_{i}+4 \tilde{\eta}^{i} \log |x|-\left(\left(1+\tilde{\eta}^{i}\right) H\left(\tilde{x}_{i}, \tilde{x}_{i}\right)+\sum_{\ell=1, \ell \neq i}^{m}\left(1+\tilde{\eta}^{\ell}\right) G\left(\tilde{x}_{i}, \tilde{x}_{\ell}\right)\right) \\
& +\mathcal{O}\left(\left|x-\tilde{x}_{i}\right|^{2}\right)+\mathcal{O}\left(\frac{\varepsilon^{2} \tau_{i}^{-2}}{\left|x-\tilde{x}_{i}\right|^{2}}\right)+\mathcal{O}\left(r_{\varepsilon, \lambda}^{2}\right) \\
= & -2 \log \tau_{i}+4 \tilde{\eta}^{i} \log r_{\varepsilon, \lambda}-\mathcal{F}_{i}\left(\tilde{x}_{i}, \tilde{x}\right)+\mathcal{O}(\varepsilon)+\mathcal{O}\left(r_{\varepsilon, \lambda}^{2}\right) \\
= & -2 \log \tau_{i}+4 \tilde{\eta}^{i} \log r_{\varepsilon, \lambda}-\mathcal{F}_{i}\left(\tilde{x}_{i}, \tilde{x}\right)+\mathcal{O}\left(r_{\varepsilon, \lambda}^{2}\right)
\end{aligned}
$$

where $\tilde{\mathbf{x}}=\left(\tilde{x}_{1}, \ldots, \tilde{x}_{m}\right)$.

Next, in (47), all functions are defined on $\partial B_{r_{\varepsilon, \lambda}}\left(\tilde{x}_{i}\right)$, but it will be convenient to solve the following equations

$$
\left(v_{\text {int }}^{i}-v_{\text {ext }}\right)\left(\tilde{x}_{i}+r_{\varepsilon, \lambda} \cdot\right)=0 \quad \text { and } \quad \partial_{r}\left(\left(v_{\text {int }}^{i}-v_{\text {ext }}\right)\left(\tilde{x}_{i}+r_{\varepsilon, \lambda} \cdot\right)\right)=0
$$

on $S^{1}$. Here, all functions are considered as functions of $y \in S^{1}$ and we have simply used the change of variables $x=\tilde{x}_{i}+r_{\varepsilon, \lambda} y$ to parameterize $\partial B_{\varepsilon_{\varepsilon, \lambda}}\left(\tilde{x}_{i}\right)$.

Since the boundary data, we have chosen satisfy (24) and (26), we can decompose

$$
\varphi^{i}=\varphi_{0}^{i}+\varphi_{1}^{i}+\varphi^{i, \perp} \quad \text { and } \quad \tilde{\varphi}^{i}=\tilde{\varphi}_{0}^{i}+\tilde{\varphi}_{1}^{i}+\tilde{\varphi}^{i, \perp}
$$


where $\varphi_{0}^{i}, \tilde{\varphi}_{0}^{i} \in \mathbb{E}_{0}=\mathbb{R}$ are constant functions on $S^{1}, \varphi_{1}^{i}, \tilde{\varphi}_{1}^{i}$ belong to $\mathbb{E}_{1}=\operatorname{ker}\left(\Delta_{S^{1}}+1\right)=\operatorname{Span}\left\{e_{1}, e_{2}\right\}$ and where $\varphi^{i, \perp}, \tilde{\varphi}^{i, \perp}$ are $L^{2}\left(S^{1}\right)$ orthogonal to $\mathbb{E}_{0}$ and $\mathbb{E}_{1}$. Projecting the equations (51) over $\mathbb{E}_{0}$ will yield the system

$$
\left\{\begin{aligned}
-2 \log \tau_{i}+4 \tilde{\eta}^{i} \log r_{\varepsilon, \lambda}-\mathcal{F}_{i}\left(\tilde{x}_{i}, \tilde{\mathbf{x}}\right)+\mathcal{O}\left(r_{\varepsilon, \lambda}^{2}\right) & =0 \\
4 \tilde{\eta}^{i}+\mathcal{O}\left(r_{\varepsilon, \lambda}^{2}\right) & =0 .
\end{aligned}\right.
$$

Let us comment briefly on how these equations are obtained. They simply come from (51) when expansions (48) and (49) are used, together with the expression of $H^{i}$ and $H^{e}$ given in Lemma 2 and Lemma 3, and also the estimates (45) and (46). The system (52) can be readily simplified into

$$
\frac{1}{\log r_{\varepsilon, \lambda}}\left[2 \log \tau_{i}+\mathcal{F}_{i}\left(\tilde{x}_{i}, \tilde{\mathbf{x}}\right)\right]=\mathcal{O}\left(r_{\varepsilon, \lambda}^{2}\right) \quad \text { and } \quad \tilde{\eta}^{i}=\mathcal{O}\left(r_{\varepsilon, \lambda}^{2}\right) \text {. }
$$

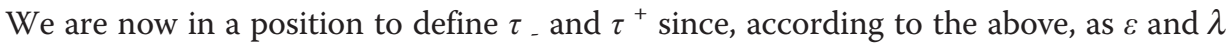
tend to 0 we expect that $\tilde{x}_{i}$ will converge to $x_{i}$ and that $\tau_{i}$ will converge to $\tau_{i}^{*}$ satisfying

$$
2 \log \tau_{i}^{*}=-\mathcal{F}_{i}\left(x_{i}, \mathbf{x}\right)
$$

and hence, it is enough to choose $\tau$ - and $\tau^{+}$in such a way that

$$
2 \log \left(\tau_{-}\right)<-\sup _{i} \mathcal{F}_{i}\left(x_{i}, \mathbf{x}\right) \leq-\inf _{i} \mathcal{F}_{i}\left(x_{i}, \mathbf{x}\right)<2 \log \left(\tau^{+}\right) .
$$

We now consider the $L^{2}$-projection of (51) over $\mathbb{E}_{1}$. Given a smooth function $f$ defined in $\Omega$, we identify its gradient $\nabla f=\left(\partial_{x_{1}} f, \partial_{x_{2}} f\right)$ with the element of $\mathbb{E}_{1}$

$$
\bar{\nabla} f=\sum_{i=1}^{2} \partial_{x_{i}} f e_{i}
$$

With these notations in mind, we obtain the equations

$$
\bar{\nabla} \mathcal{F}_{i}\left(\tilde{x}_{i}, \tilde{\mathbf{x}}\right)=\mathcal{O}\left(r_{\varepsilon, \lambda}^{2}\right) \quad \text { and } \quad \varphi_{1}^{i}=\mathcal{O}\left(r_{\varepsilon, \lambda}^{2}\right)
$$

Finally, we consider the $L^{2}$-projection onto $L^{2}\left(S^{1}\right)^{\perp}$. This yields the system

$$
\left\{\begin{array}{r}
\varphi^{i, \perp}-\tilde{\varphi}^{i, \perp}+\mathcal{O}\left(r_{\varepsilon, \lambda}^{2}\right)=0 \\
\partial_{r}\left(H^{i, \perp}-H^{e, \perp}\right)+\mathcal{O}\left(r_{\varepsilon, \lambda}^{2}\right)=0 .
\end{array}\right.
$$

Thanks to the result of Lemma 4, this last system can be re-written as

$$
\varphi^{i, \perp}=\mathcal{O}\left(r_{\varepsilon, \lambda}^{2}\right) \quad \text { and } \quad \tilde{\varphi}^{i, \perp}=\mathcal{O}\left(r_{\varepsilon, \lambda}^{2}\right) .
$$

If we define the parameters $\mathbf{t}=\left(t_{i}\right) \in \mathbb{R}^{m}$ by

$$
t_{i}=\frac{1}{\log r_{\varepsilon, \lambda}}\left[2 \log \tau_{i}+\mathcal{F}_{i}\left(\tilde{x}_{i}, \tilde{x}\right)\right], \quad \text { for } i=1, \cdots, m
$$

then, the system we have to solve reads

$$
\left(\mathbf{t}, \tilde{\eta}, \varphi_{0}, \tilde{\varphi}_{0}, \varphi_{1}, \tilde{\varphi}_{1}, \bar{\nabla} \mathcal{F}(\tilde{\mathbf{x}}, \mathbf{x}), \varphi^{\perp}, \tilde{\varphi}^{\perp}\right)=\mathcal{O}\left(r_{\varepsilon, \lambda}^{2}\right),
$$

where as usual, the terms $\mathcal{O}\left(r_{\varepsilon, \lambda}^{2}\right)$ depend nonlinearly on all the variables on the left side, but is bounded (in the appropriate norm) by a constant (independent of $\varepsilon$ and $\lambda$ ) time $r_{\varepsilon, \lambda}^{2}$, provide $\varepsilon \in\left(0, \varepsilon_{\kappa}\right)$ and $\lambda \in\left(0, \lambda_{\kappa}\right)$. Then, the nonlinear mapping which appears on the right-hand side of (55) is continuous and compact. In addition, reducing $\varepsilon_{\kappa}$ and $\lambda_{\kappa}$ if necessary, this nonlinear mapping sends the ball of radius $\kappa r_{\varepsilon, \lambda}^{2}$ (for 
the natural product norm) into itself, provided $\kappa$ is fixed large enough. Applying Schauder's fixed Theorem in the ball of radius $\kappa r_{\varepsilon, \lambda}^{2}$ in the product space where the entries live yields the existence of a solution of Eq. (55) and this completes the proof of our Theorem 1.

\section{Acknowledgements}

The authors extend their appreciation to the Deanship of Scientific Research at King Saud University for funding the work through the research group project No RGP-VPP-087.

\section{Author details}

'Department of Mathematics, College of Science, King Saud University, P.O. Box 2455, Riyadh 11451, Saudi Arabia

2Département de Mathématiques, Faculté des Sciences de Tunis Campus Universitaire, 2092 Tunis, Tunisia

\section{Authors' contribution}

The authors declare that the work was realized in collaboration with same responsibility. All authors read and approved the final manuscript.

\section{Competing interests}

The authors declare that they have no competing interests.

Received: 22 March 2011 Accepted: 12 August 2011 Published: 12 August 2011

\section{References}

1. Ren, X, Wei, J: On a two-dimensional elliptic problem with large exponent in nonlinearity. Trans Am Math Soc. 343 , 749-763 (1994)

2. Esposito, P, Musso, M, Pistoia, A: Concentrating solutions for a planar problem involving nonlinearities with large exponent. J Diff Eqns. 227, 29-68 (2006)

3. Baraket, S, Ben Omrane, I, Ouni, T: Singular limits solutions for 2-dimensional elliptic problem involving exponential nonlinearities with non linear gradient term. Nonlinear Differ Equ Appl. 18, 59-78 (2011)

4. Baraket, S, Ye, D: Singular limit solutions for two-dimensional elliptic problems with exponentionally dominated nonlinearity. Chin Ann Math Ser B. 22, 287-296 (2001)

5. Liouville, J: Sur l'équation aux différences partielles. J Math. 18, 17-72 (1853)

6. Tarantello, G: Multiple condensate solutions for the Chern-Simons-Higgs theory. J Math Phys. 37, 3769-3796 (1996)

7. Suzuki, T: Two dimensional Emden-Fowler Equation with Exponential Nonlinearity. Nonlinear Diffusion Equations and Their Equilibrium States Birkäuser. 3, 493-512 (1992)

8. Nangasaki, K, Suzuki, T: Asymptotic analysis for two-dimensional elliptic eigenvalue problems with exponentially dominated nonlinearities. Asymptotic Anal. 3, 173-188 (1990)

9. Ye, D: Une remarque sur le comportement asymptotique des solutions de $-\Delta u=\lambda f(u)$. C R Acad Sci Paris I. 325, 1279-1282 (1997)

10. Weston, $\mathrm{VH}$ : On the asymptotique solution of a partial differential equation with exponential nonlinearity. SIAM J Math. 9, 1030-1053 (1978)

11. Baraket, S, Pacard, F: Construction of singular limits for a semilinear elliptic equation in dimension 2. Calc Var Partial Differ Equ. 6, 1-38 (1998)

12. Esposito, P, Grossi, M, Pistoia, A: On the existence of Blowing-up solutions for a mean field equation. Ann I H Poincaré -AN. 22, 227-257 (2005)

13. Del Pino, M, Kowalczyk, M, Musso, M: Singular limits in Liouville-type equations. Calc Var Partial Differ Equ. $24,47-87$ (2005)

14. Wei, J, Ye, D, Zhou, F: Bubbling solutions for an anisotropic Emden-Fowler equation. Calc Var Partial Differ Equ. 28, 217-247 (2007)

15. Wei, J, Ye, D, Zhou, F: Analysis of boundary bubbling solutions for an anisotropic Emden-Fowler equation. Ann I H Poincaré AN. 25, 425-447 (2008)

16. Ye, D, Zhou, F: A generalized two dimensional Emden-Fowler equation with exponential nonlinearity. Calc Var Partial Differ Equ. 13, 141-158 (2001)

17. Wente, HC: Counter example to a conjecture of H. Hopf. Pacific J Math. 121, 193-243 (1986)

18. Baraket, S, Dammak, M, Ouni, T, Pacard, F: Singular limits for a 4-dimensional semilinear elliptic problem with exponential nonlinearity. Ann I H Poincaré AN. 24, 875-895 (2007)

19. Dammak, M, Ouni, T: Singular limits for 4-dimensional semilinear elliptic problem with exponential nonlinearity adding a singular source term given by Dirac masses. Differ Int Equ. 11-12, 1019-1036 (2008)

20. Clapp, M, Munoz, C, Musso, M: Singular limits for the bi-Laplacian operator with exponential nonlinearity in $\mathbb{Z}^{4}$. Ann I H Poincaré AN. 25, 1015-1041 (2008)

21. Baraket, S, Ben Omrane, I, Ouni, T, Trabelsi, N: Singular limits solutions for 2-dimensional elliptic problem with exponentially dominated nonlinearity and singular data. Communications in Contemporary Mathematics 2. 13(4), 129 (2011)

doi:10.1186/1687-2770-2011-10

Cite this article as: Baraket et al: Singular limiting solutions for elliptic problem involving exponentially dominated nonlinearity and convection term. Boundary Value Problems 2011 2011:10. 